\title{
OBTENÇÃO DE FARINHAS MISTAS PRÉ-GELATINIZADAS A PARTIR DE ARROZ E BAGAÇO DE JABUTICABA: EFEITO DAS VARIÁVEIS DE EXTRUSÃO NAS PROPRIEDADES DE PASTA
}

\author{
DIEGO PALMIRO RAMIREZ ASCHERI ${ }^{*}$ \\ CRISTINA TRISTÃO DE ANDRADE ${ }^{* *}$ \\ CARLOS WANDERLEI PILER DE CARVALHO*** \\ JOSÉ LUIS RAMÍREZ ASCHERI***
}

\begin{abstract}
No presente trabalho estudou-se o efeito da combinação da velocidade de rotação do parafuso $(65,9-234,1 \mathrm{rpm})$, da temperatura na terceira zona do barril do extrusor $(99,5-$ $200,1^{\circ} \mathrm{C}$ ) e da formulação de farinha de bagaço de jabuticaba $(11,59-28,41 \%)$, utilizada como fonte de fibra, nas propriedades de pasta de farinhas mistas pré-gelatinizadas de arroz polido e bagaço de jabuticaba. Foram determinadas a fibra detergente ácido (FDA) e a fibra detergente neutro (FDN) das farinhas cruas e suas misturas. As propriedades de pasta foram determinadas em Analisador Rápido de Viscosidade (RVA). A farinha de bagaço de jabuticaba (FBJ) apresentou altos valores de FDA $(21,91 \%)$ e de FDN $(45,47 \%)$ o que contribuiu para aumentar o teor de fibra alimentar das misturas extrusadas (FME). As amostras estudadas apresentaram perfis diferentes de viscosidade de pasta com valores mínimos para FBJ, valores máximos para FAP e valores intermediários para FME. A viscosidade inicial a $25^{\circ} \mathrm{C}$ foi fortemente influenciada pela formulação e pela velocidade de rotação do parafuso, seguidas pela temperatura. A viscosidade máxima a $95^{\circ} \mathrm{C}$ (VMAX) e a viscosidade final (VFINAL) foram intensamente influenciadas pela formulação e temperatura, seguidas pela velocidade de rotação do parafuso. Ambas aumentaram com a elevação da temperatura e diminuíram com o incremento da porcentagem de FBJ na mistura, bem como pelo aumento da velocidade de rotação do parafuso.
\end{abstract}

PALAVRAS-CHAVE: EXTRUSÃO TERMOPLÁSTICA; FARINHA DE ARROZ; FIBRA ALIMENTAR; JABUTICABA.

* Engenheiro de Alimentos, Doutor em Engenharia de Alimentos, Professor concursado da Universidade Estadual de Goiás (UEG), Anápolis, GO (e-mail: ascheridpr@uol.com.br).

** Engenheira Química, Doutora em Ciência e Tecnologia de Polímeros, Professora adjunta da Universidade Federal do Rio de Janeiro, Ilha do Fundão, Rio de Janeiro, RJ (e-mail: ctandrade@ima.ufr..br).

*** Engenheiro Agrônomo, Doutor em Ciência dos Alimentos, Pesquisador da Embrapa Agroindústria de Alimentos, Guaratiba, RJ (e-mail: cwpiler@ctaa.embrapa.br).

**** Engenheiro de Alimentos, Doutor em Tecnologia dos Alimentos, Pesquisador da Embrapa Agroindústria de Alimentos (e-mail: ascheri@ctaa.embrapa.br). 


\section{INTRODUÇÃO}

A extrusão constitui processo contínuo, versátil e muito utilizado na tecnologia de transformação de alimentos. Nos alimentos extrusados ocorrem modificações químicas e mudanças estruturais como a gelatinização de amidos (AKDOGAN, 1999, VAN DEN EINDE et al., 2005), a desnaturação de proteínas (GUY, 2001a), a formação de complexos entre amilose e lipídios (HSIEH e HUFF, 1997), reações de condensação entre proteína-açúcar (reação de Maillard) (AMES, et al. 1998; GUY, 2001b; IWE et al., 2004), reações de degradação de pigmentos e vitaminas, (ILO e BERGHOFER, 1999), perda de compostos voláteis (BHANDARI, D'ARCY e YOUNG, 2001) e outras.

O processo de extrusão tem revolucionado os métodos convencionais no preparo de cereais matinais, snacks, amidos e farinhas prégelatinizadas (KOKINI, HO, KARWE, 1992, ØSTERGÅRD, BJÖRK e VAINIONPÅÅ, 1989). Esses produtos geralmente são elaborados a base de cereais (como o milho e o arroz) e de amidos de raízes e tubérculos como a mandioca e a batata (BALAGOPALAN, 2002; BOONYASIRIKOOL e CHARUNUCH, 2000; CHEYNE, BARNES e WILSON, 2005; CHUANG e YEH, 2004; DING et al. 2005).

As farinhas pré-gelatinizadas por extrusão a base de arroz polido são boas fontes energéticas, porém pobres em fibra alimentar. A American Association of Cereal Chemists definiu fibra alimentar como a parte remanescente da porção comestível, ou de carboidratos análogos que são resistentes à digestão e à absorção no intestino delgado humano, com fermentação completa ou parcial no intestino grosso. A fibra alimentar inclui polissacarídios, oligossacarídios, lignina e outras substâncias associadas. A fibra alimentar promove efeitos fisiológicos benéficos ao ser humano, como os laxativos, atenuação do colesterol sangüíneo e/ou da glicose sangüínea (CAMIRE, 2001).

A presença de fibra em produtos extrusados a base de arroz pode abreviar o tempo de trânsito intestinal do produto extrusado, aumentar a velocidade de absorção intestinal da glicose, diminuir os níveis de colesterol sangüíneo e reduzir o conteúdo de calorias ingeridas. Essas propriedades, segundo Calixto apud BOTELHO, CONCEIÇÃO e CARVALHO (2002), torna as fibras em adequado regulador intestinal. 
As fibras são ainda fatores de importância em regimes dietéticos para a prevenção ou tratamento de diabetes, problemas de hipercolesterolemia e obesidade. Ao contrário, a falta de fibra na dieta pode estar relacionada com o desenvolvimento de câncer de cólon e outros distúrbios gastrointestinais.

Tendo em conta o aumento da demanda de alimentos com alto conteúdo de fibra alimentar, que tem propiciado o desenvolvimento de procedimentos para obter melhores concentrados de fibra, há necessidade de se buscar novas fontes de fibras que possam substituir parte das calorias fornecidas pelas farinhas pré-gelatinizadas. Acreditase que o bagaço de jabuticaba (subproduto da indústria de licores e sucos de jabuticaba) possa fornecer além de fibra alimentar, sais minerais e proteínas remanescentes do agente biológico utilizado para a fermentação do suco de jabuticaba.

A principal preocupação durante o processo de extrusão de farinhas mistas de arroz polido e bagaço de jabuticaba envolve a combinação dos parâmetros de extrusão e da proporção adequada de bagaço utilizado para não prejudicar as propriedades físico-químicas das farinhas pré-gelatinizadas, ou mesmo fornecer propriedades funcionais aplicáveis à indústria de alimentos.

As propriedades físico-químicas de diferentes materiais amiláceos extrusados tem sido extensivamente pesquisados em função dos parâmetros do processo de extrusão. CHUANG e YEH (2004) estudaram o efeito do perfil do parafuso no tempo de residência do amido da farinha de arroz mediante extrusor mono-rosca. Observaram que o grau de gelatinização do amido da farinha de arroz tende a elevar-se com o aumento do tempo de residência da farinha no interior do barril provocado pelo tipo de configuração do parafuso. DING et al. (2005) pesquisaram o efeito das condições de extrusão nas propriedades físico-químicas e nas características sensoriais de snacks expandidos a base de arroz. Verificaram que a velocidade do parafuso (180-320rpm) não exerceu efeito significativo sobre essas propriedades. Entretanto, CHIANG e JOHNSON (1977) afirmaram que o grau de gelatinização do amido diminuiu com alta umidade e o aumento da velocidade do parafuso. 
A temperatura utilizada no processo de extrusão exerce papel importante nas mudanças das propriedades físico-químicas dos produtos extrusados. LAWTON, HENDERSON e DERLATSKA (1972) consideraram, além da umidade inicial da matéria-prima, a temperatura como a variável de maior efeito na gelatinização do amido. VAN LENGERICH (1990) observou que amidos degradados são compostos de moléculas com baixo peso molecular em decorrência da dextrinização do amido. Esse fenômeno, usualmente ocorre em temperaturas de extrusão maiores do que $160^{\circ} \mathrm{C}$ (CHIANG e JOHNSON, 1977; COLONNA et al. 1984; LAI e KOKINI 1992; LI, CAMPANELLA e HARDACRE, 2004; VERGNES, DELLA VALLE e TAYEB, 1993). Entretanto, LI, CAMPANELLA e HARDACRE (2004) observaram maior grau de gelatinização do amido de milho quando extrusado a $130^{\circ} \mathrm{C}$. SACCHETTI et al. (2004) reforçaram essa observação, porém usando amido de arroz extrusado em temperatura de $120^{\circ} \mathrm{C}$.

Vários autores estudaram o efeito da formulação de misturas de alimentos nas características dos produtos extrusados. A inclusão de ingredientes no material amiláceo a ser extrusado influencia as características físico-químicas do produto final, podendo diminuir a gelatinização do amido por não oferecerem material viscoso suficiente (MAGA e FAPOJUWO, 1996 e 1988). CARVALHO, ASCHERI e CAL-VIDAL (2002) estudando a influência da mistura de farinha de trigo, arroz e banana, e FERNANDES et al. (2002), em canjica e soja, observaram que a formulação das farinhas do produto a ser extrusado influencia a gelatinização do amido. Também afirmaram que quanto maior a proporção de material não-amiláceo, menor o grau de gelatinização do amido do produto extrusado em decorrência da diminuição da proporção de amido.

No presente trabalho estudou-se o efeito da combinação da velocidade de rotação do parafuso $(65,9-234,1 \mathrm{rpm})$, da temperatura na terceira zona do barril do extrusor $\left(99,5-200,1^{\circ} \mathrm{C}\right)$ e da formulação de farinha de bagaço de jabuticaba (11,59-28,41\%), utilizada como fonte de fibra, nas propriedades de pasta de farinhas mistas pré-gelatinizadas de arroz polido e bagaço de jabuticaba. 


\section{MATERIAL E MÉTODOS}

\subsection{MATÉRIA-PRIMA}

O bagaço de jabuticaba, subproduto da indústria de licores e sucos de jabuticaba, foi fornecido pela Fazenda Jabuticabal (município de Nova Fátima - GO). Aproximadamente $170 \mathrm{~kg}$ de bagaço foram coletados em sacos de polietileno e fechados hermeticamente para minimizar as perdas de umidade. O bagaço foi coletado logo após o esgotamento total do suco fermentado, mediante prensagem ao final do processo de fermentação do suco de jabuticaba.

Após a coleta, o bagaço de jabuticaba foi desidratado e moído no Laboratório de Alimentos da Universidade Estadual de Goiás (Anápolis). Distribui-se o bagaço uniformemente em bandejas, colocando-as em estufa com recirculação de ar forçado (marca Tecnal) a $60^{\circ} \mathrm{C}$ até atingir $7,0 \%$ de umidade. Moeu-se o bagaço desidratado em moinho de bolas, obtendo-se a farinha de bagaço de jabuticaba (FBJ).

O arroz (Oryza sativa, L.) polido, marca Blue-Platina, foi adquirido no comércio da cidade do Rio de Janeiro em sacos de $5 \mathrm{~kg}$. O arroz com umidade inicial de $12,63 \%$ foi moído em moinho de rolo (tipo QU-J BRABENDER), obtendo-se a farinha de arroz polido (FAP).

\subsection{PROCEDIMENTO EXPERIMENTAL}

\subsubsection{Fibra em detergente neutro e em detergente ácido}

Foram determinados os teores de fibra em detergente neutro (FDN) e em detergente ácido (FDA) do bagaço de jabuticaba de acordo com a metodologia descrita por SOUZA et al. (1999).

\subsubsection{Preparo e acondicionamento das farinhas cruas}

As farinhas cruas foram condicionadas manualmente em sacos plásticos de polietileno, com umidade de $16 \%$, conforme quantidade de massa seca das farinhas pré-estabelecida no delineamento experimental, obtendo-se as farinhas mistas. O teor de umidade inicial das farinhas mistas (que variou de 10,70 a $11,65 \%$ ) foi determinado 
pelo método 44-16 A da AACC (1995) em estufa a $130^{\circ} \mathrm{C}$ por uma hora. Calculou-se a quantidade de água (que variou de 51,2 a $63,1 \mathrm{~mL} /$ $1000 \mathrm{~g}$ de farinha) adicionada às misturas por meio da seguinte fórmula:

$$
Q_{a}=\frac{\left(U_{f}-U_{i}\right)}{100-U_{f}} \cdot m_{a}
$$

Na qual:

$\mathrm{Q}_{\mathrm{a}}=$ quantidade de água a ser adicionada, em mililitros;

$\mathrm{U}_{\mathrm{f}}=$ umidade final da amostra estabelecida a $16 \%$;

$\mathrm{U}_{\mathrm{i}}=$ umidade inicial da amostra;

$\mathrm{m}_{\mathrm{a}}=$ massa da amostra, em gramas .

As amostras, após homogeneização, foram acondicionadas em sacos plásticos e armazenadas sob refrigeração por 24 horas para se obter distribuição e absorção mais uniforme da água.

\subsection{EXTRUSÃO}

Utilizou-se extrusor da marca BRABENDER (modelo 20DN) de parafuso único, equipado com matriz circular de $3 \mathrm{~mm}$, taxa de compressão do parafuso único de $3: 1$, taxa de alimentação $5 \mathrm{~kg} / \mathrm{h}$, sendo as temperaturas das zonas 1 e 2 mantidas constantes a 50 e $100^{\circ} \mathrm{C}$, respectivamente. A velocidade do parafuso e a temperatura variaram de acordo com o delineamento experimental descrito na Tabela 1. Quando o equilíbrio do processo (monitorado pela visualização da variação do torque pelo equipamento) foi atingido cortou-se o material extrusado, manualmente, em tamanho aproximado de $30 \mathrm{~cm}$ de comprimento, secando-o em estufa com circulação de ar a 75드 até umidade final entre 3 e $7 \%$. As amostras foram moídas em moinho de disco (PERTEM) e de rolos (BRABENDER JUNIOR), obtendo-se farinhas mistas extrusadas (pré-gelatinizadas, FME). Após moagem, as FME foram acondicionadas em sacos plásticos devidamente etiquetados e armazenados em temperatura ambiente.

\subsection{PLANEJAMENTO EXPERIMENTAL}

Para estudar o efeito da velocidade do parafuso $(R)$, da temperatura de aquecimento da última zona $(\mathrm{T})$ e da proporção de FBJ sobre as 
propriedades de pasta das FME usou-se delineamento central composto com duas repetições no ponto central e seis axiais. As variáveis foram estabelecidas com três níveis codificados $-1,0,+1$ (BOX, HUNTER e HUNTER, 1978). Esse delineamento apresenta seis níveis de variáveis axiais codificados como $-\alpha e+\alpha$. O valor de $\alpha$ é função do número de variáveis independentes, sendo definido pela seguinte equação:

$$
\alpha=\left(2^{k}\right)^{1 / 4}=\left(2^{3}\right)^{1 / 4}=1,628
$$

O delineamento estatístico requer número mínimo de ensaios experimentais. Foram usados 16 ensaios, sendo oito fatoriais (combinações dos níveis $-1 e+1$ ), seis axiais (uma variável no nível $\pm \alpha$ e outra no nível 0 ) e dois centrais servindo como estimativa do erro experimental e para determinar a precisão da equação polinomial (COCHRAN e COX, 1964).

$\mathrm{Na}$ Tabela 1 apresentam-se os valores codificados e reais do delineamento experimental em estrela para três variáveis independentes e cinco níveis de variação. As respostas $(\mathrm{Y})$ ou variáveis dependentes estudadas foram: viscosidade inicial a $25^{\circ} \mathrm{C}$ (VINIC), viscosidade máxima a $95^{\circ} \mathrm{C}(\mathrm{VMAX})$ e viscosidade final (VFIN), quantificados em centipoise $(\mathrm{cP})$.

\subsection{VISCOSIDADE DE PASTA}

Determinou-se a viscosidade de pasta (VP) em Analisador Rápido de Viscosidade (RVA da Newport Scientific), seguindo basicamente a metodologia de materiais extrusados do seu manual. É importante considerar alguns aspectos de preparação das amostras, como o tipo de moinho e tamanho de partículas do material a ser analisado no RVA. As amostras moídas foram peneiradas e a fração entre as peneiras 250 e $106 \mu \mathrm{m}$ analisada em duplicata. A VP foi expressa em $\mathrm{CP}$.

Para a análise no RVA, $3 \mathrm{~g}$ de farinha extrusada com umidade corrigida para $14 \%$ (em base úmida) foi adicionada de água destilada até peso final de $28 \mathrm{~g}$. Aumentou-se a temperatura inicial de $25^{\circ} \mathrm{C}$ gradualmente para $95^{\circ} \mathrm{C}$, com taxa de aquecimento de $14^{\circ} \mathrm{C} /$ minuto, permanecendo 
constante nessa temperatura por 3 minutos. O resfriamento também ocorreu gradualmente até atingir a temperatura final de $25^{\circ} \mathrm{C} . \mathrm{Na}$ interpretação dos viscoamilogramas foram considerados os seguintes parâmetros:

a) Viscosidade inicial (VINIC) da pasta a $25^{\circ} \mathrm{C}$ ("a frio") = valor máximo de viscosidade (em cP) no início do ciclo de aquecimento, que depende (principalmente) do grau de gelatinização dos grânulos de amido e da magnitude da sua quebra molecular durante o processo de extrusão.

b) Viscosidade máxima $(\mathrm{VMAX})=$ valor da viscosidade $(\mathrm{em} \mathrm{cP})$ no ponto máximo da curva, obtido durante o ciclo de aquecimento.

c) Viscosidade final (VFIN) a $25^{\circ} \mathrm{C}$ (ciclo de resfriamento) $=$ valor observado quando os elementos presentes na pasta começam a se associar ou retrogradar, aumentando a viscosidade.

TABELA 1 - VARIÁVEIS INDEPENDENTES COM DIFERENTES NÍVEIS E MATRIZ DOS ENSAIOS PARA O PLANEJAMENTO FATORIAL EM ESTRELA COM AS VARIÁVEIS NAS UNIDADES REAIS E CODIFICADAS

\begin{tabular}{|c|c|c|c|c|c|c|}
\hline \multirow[b]{2}{*}{ Variável } & & \multicolumn{5}{|c|}{ Nivel } \\
\hline & & $-1,682$ & .1 & 0 & +1 & $+1,682$ \\
\hline $\mathrm{R}(\mathrm{rpm})$ & & 65,90 & 100 & 150 & 200 & 234,10 \\
\hline $\mathrm{T}\left({ }^{\circ} \mathrm{C}\right)$ & & 99,54 & 120 & 150 & 180 & 200,46 \\
\hline Fo $(\%)$ & & 11,59 & 15 & 20 & 25 & 28,41 \\
\hline Ensaio & $X_{1}$ & $X_{2}$ & $X_{3}$ & $\mathrm{R}$ (rpm) & $\mathrm{T}\left({ }^{\circ} \mathrm{C}\right)$ & Fo $(\%)$ \\
\hline$E_{1}$ & .1 & .1 & .1 & 100 & 120 & 15 \\
\hline$E_{2}$ & -1 & -1 & 1 & 100 & 120 & 25 \\
\hline$E_{3}$ & -1 & 1 & .1 & 100 & 180 & 15 \\
\hline$E_{4}$ & -1 & 1 & 1 & 100 & 180 & 25 \\
\hline$E_{5}$ & 1 & -1 & .1 & 200 & 120 & 15 \\
\hline$E_{6}$ & 1 & -1 & 1 & 200 & 120 & 25 \\
\hline$E_{7}$ & 1 & 1 & -1 & 200 & 180 & 15 \\
\hline$E_{8}$ & 1 & 1 & 1 & 200 & 180 & 25 \\
\hline$E_{9}$ & $-1,682$ & 0 & 0 & 65,91 & 150 & 20 \\
\hline$E_{10}$ & 1,682 & 0 & 0 & 234,09 & 150 & 20 \\
\hline$E_{11}$ & 0 & $\cdot 1,682$ & 0 & 150 & 99,55 & 20 \\
\hline$E_{12}$ & 0 & 1,682 & 0 & 150 & 200,45 & 20 \\
\hline$E_{13}$ & 0 & 0 & $-1,682$ & 150 & 150 & 11,59 \\
\hline$E_{14}$ & 0 & 0 & 1,682 & 150 & 150 & 28,41 \\
\hline$E_{15}$ & 0 & 0 & 0 & 150 & 150 & 20 \\
\hline$E_{16}$ & 0 & 0 & 0 & 150 & 150 & 20 \\
\hline
\end{tabular}

$\mathrm{X}_{1}=$ Rotação do parafuso, $\mathrm{R}(\mathrm{rpm}) ; \mathrm{X}_{2}=$ Temperatura, $\mathrm{T}\left({ }^{\circ} \mathrm{C}\right) ; \mathrm{X}_{3}=$ Formulação, Fo (\%). 


\subsection{ANÁLISE ESTATÍSTICA DOS RESULTADOS}

A fim de verificar se houve diferença entre as médias das variáveis resposta da Tabela 1 aplicou-se a ANOVA fator único e o teste de Tukey ao nível de $5 \%$ de probabilidade (PIMENTEL-GOMES, 2000). Usando a ANOVA (ao nível de $5 \%$ de probabilidade) aplicou-se equação de segunda ordem para estabelecer o modelo matemático preditivo das variáveis resposta, cuja equação geral foi:

$Y=b_{0}+b_{1} X_{1}+b_{2} X_{2}+b_{3} X_{3}+b_{11} X_{1}^{2}+b_{22} X_{2}{ }^{2}+b_{33} X_{3}{ }^{2}+b_{12} X_{1} X_{2}$ $+b_{13} X_{1} X_{3}+b_{23} X_{2} X_{3}+\xi$

Na qual:

$Y=$ função resposta genérica;

$\mathrm{X}=$ variável codificada, obtida a partir da variável original;

$\mathrm{b}=$ coeficientes estimados pelos métodos dos mínimos quadrados;

$\xi=$ erro experimental (BOX, HUNTER e HUNTER, 1978).

Os gráficos de superfície de resposta, os coeficientes e respectivos efeitos do modelo matemático foram obtidos pela Metodologia de Superfície de Resposta (MSR) usando-se o programa Statistica for Windows (STATSOFT, 1996).

\section{RESULTADOS E DISCUSSÃO}

\subsection{TEOR DE FIBRAS}

Segundo POURCHET-CAMPOS (1988), a fração fibra alimentar é constituída pela fração insolúvel formada por celulose, algumas hemiceluloses e lignina. Já a fração solúvel contém pectinas, gomas, mucilagens e algumas hemiceluloses. A fibra detergente ácido corresponde ao somatório de celulose e lignina ao passo que a fibra detergente neutro ao conjunto de celulose, hemicelulose e lignina. A determinação de fibras em detergente ácido e em detergente neutro mostrou que a FBJ é rica em fibras insolúveis, apresentando valores de FDA e FDN de $21,91 \%$ ( \pm 0,04\%) e $45,47 \%$ ( $\pm 1,53 \%$ ) em base seca, respectivamente. $O$ teor dessas frações correspondentes às farinhas mistas dos diferentes ensaios variaram de $2,54 \%$ a $6,22 \%$ de 
FDA e de $2,57 \%$ a $12,92 \%$ de FDN. Os ensaios $E_{1}, E_{3}, E_{5}$ e $E_{7}$ apresentaram 3,29\% e 6,82\% de FDA e FDN, os ensaios $E_{2}, E_{4}, E_{6}$ e $E_{8}, 5,48 \%$ e $11,37 \%$, os ensaios $E_{9}$ a $E_{12}$, $E_{15}$ e $E_{16} 4,38 \%$ e $9,09 \%$, o ensaio $E_{13} 2,54 \%$ e $5,27 \%$ e o ensaio $E_{14} 6,22 \%$ e $12,92 \%$, respectivamente.

De acordo com DREHER (1995), o alimento com teor entre 2 a 3\% de fibra pode ser considerado boa fonte de fibra alimentar. Segundo o regulamento técnico referente à informação nutricional complementar (Portaria $\mathrm{n}^{\circ} 27$ ), o alimento pode ser considerado fonte de fibra alimentar quando apresenta no produto acabado $3 \mathrm{~g} / 100 \mathrm{~g}$ (base integral) para alimentos sólidos e $1,5 \mathrm{~g} / 100 \mathrm{~mL}$ (base integral) para líquidos, já com o dobro desse conteúdo pode ser tratado como alimento com elevado teor de fibra alimentar (BRASIL, 1998). Os teores de FDN presentes nas farinhas mistas de arroz polido e bagaço de jabuticaba permitiram considerar todas as misturas como boas fontes de fibra alimentar.

\subsection{PERFIL DE VISCOSIDADE DA PASTA}

Os perfis de viscosidade das pastas das farinhas de bagaço de jabuticaba, de arroz polido e das farinhas mistas extrusadas são apresentados nas Figuras 1 ( $a$ e b) e 2, respectivamente.

A Figura 1a mostra o perfil de viscosidade de pasta da FBJ em função do tempo e da temperatura. O ruído observado deve-se à sensibilidade do equipamento, sendo evidenciado pela baixa viscosidade de pasta dessa amostra. Inicialmente, a viscosidade de $36 \mathrm{cP}$ sofreu queda durante os cinco primeiros minutos até atingir o valor mínimo de $23 \mathrm{cP}$, permanecendo constante até os 8 min seguintes com a temperatura aumentando de $39^{\circ} \mathrm{C}$ para $82^{\circ} \mathrm{C}$. Esse perfil evidencidou que a viscosidade de suspensões aquosas de FBJ é instável quando submetida à taxa de cisalhamento em temperatura ambiente, provavelmente pela elevada concentração de fibras insolúveis contida na farinha. Segundo ANTONIO (2004) e TUNGLAND e MEYER (2002), os constituintes insolúveis da fibra alimentar têm baixa capacidade de absorção de água e conferem baixa viscosidade às suspensões aquosas por eles formados. A viscosidade inicial pode ser devida à presença de fibras solúveis em pequenas concentrações, tais como hemicelulose, pectinas, taninos, glucanos, ácido fítico, gomas, inulina e outros (GARCIA-LUNA, 2003). 


\section{FIGURA 1 - PERFIL DE VISCOSIDADE: (a) DA FARINHA DE BAGAÇO DE JABUTICABA (FBJ) E (b) DA FARINHA DE ARROZ POLIDO (FAP)}
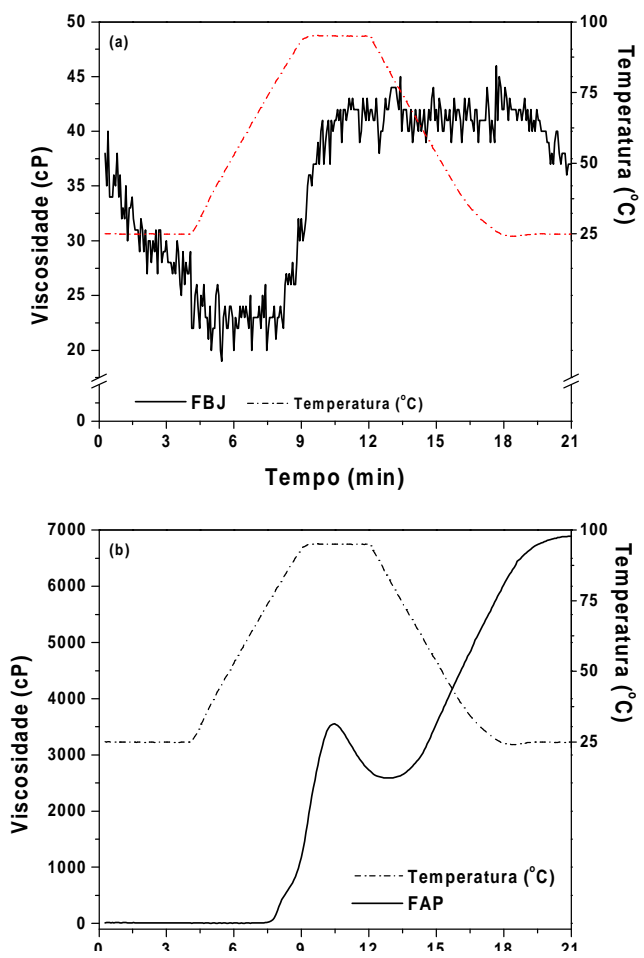

A viscosidade elevou-se até o máximo de $41 \mathrm{cP}$ a $95^{\circ} \mathrm{C}$ aos $10 \mathrm{~min}$, permaneceu constante ao longo do ciclo de resfriamento de $19 \mathrm{~min}$, seguido de queda até o final do experimento $(36 \mathrm{cP})$. O aumento da viscosidade com a elevação da temperatura ocorre pela perda parcial das pontes de hidrogênio que unem as diferentes frações presentes na FBJ. Isso origina sítios hidrofílicos que se ligam a moléculas de água, aumentando a solubilidade da fibra alimentar e a viscosidade da suspensão aquosa que retorna ao estado original em temperatura ambiente $\left(25^{\circ} \mathrm{C}\right)$.

Segundo KARAPANTSIOS, SAKONIDOU e RAPHAELIDES (2002), a 
principal conseqüência da aplicação do calor numa suspensão aquosa de produtos amiláceos é a gelatinização dos grânulos de amido. Para AKDOGAN (1996), além da gelatinização, as macromoléculas do amido podem sofrer degradação devido aos tratamentos térmico e mecânico empregados.

\section{FIGURA 2 - DIFERENTES PERFIS DE VISCOSIDADE DAS FARINHAS MISTAS EXTRUSADAS}

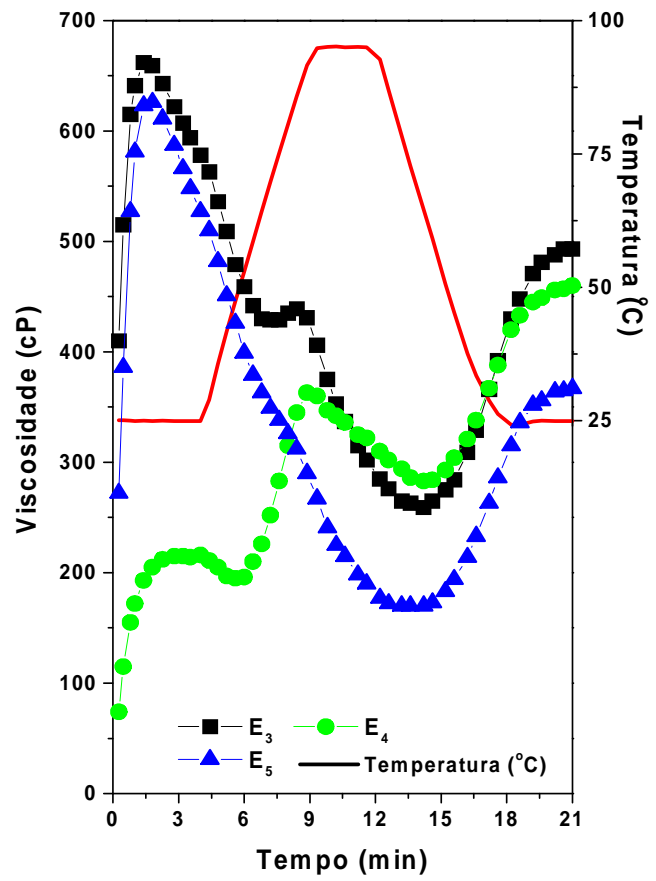

A gelatinização é a combinação do evento de fusão da porção cristalina do grânulo de amido e da transição vítrea da porção amorfa do grânulo. O grânulo de amido não é solúvel em água fria, mas aquecido em meio aquoso absorve água e intumesce. Inicialmente o entumescimento é reversível, tornando-se irreversível conforme o 
aumento da temperatura que rompe as pontes de hidrogênio e permite a incorporação de água pelo amido. Tal incorporação aumenta a separação entre as cadeias e a aleatoriedade, diminuindo o número e o tamanho das regiões cristalinas e a perda da birrefringência. $O$ rompimento dos grânulos libera a amilose, o que contribui para o aumento da viscosidade da suspensão (FUKUOKA, OHTA e WATANABE, 2002; MITCHELL et al., 1997; MATUDA, 2004; THIRÉ, SIMÃO e ANDRADE, 2003).

Ao ultrapassar a temperatura de gelatinização do amido, a suspensão se solubiliza e a viscosidade começa a diminuir. O gel formado quando resfriado tende a reconstruir estrutura mais rígida pela agregação das cadeias de amilose, formando duplas hélices cristalinas estabilizadas por pontes de hidrogênio. Isso resulta em maior perda de água do sistema e endurecimento do produto final, fenômeno chamado retrogradação (LOBO e SILVA, 2003; MUNHOZ, WEBER e CHANG, 2004).

O perfil de RVA da FAP (Figura 1b) mostrou as modificações dos grânulos de amido do arroz polido em função do tempo e da temperatura. O pico de viscosidade dos grânulos de amido com propriedades de expansão/ruptura mais uniformes, durante o tratamento hidrotérmico assume formato agudo (FENIMAN, 2004). Nos primeiros 8 minutos os grânulos de amido não sofreram modificação e a viscosidade mostrou-se muito baixa ( $\mathrm{VINIC}=28,0 \mathrm{cP}, \mathrm{T}=75^{\circ} \mathrm{C}$ ). Após esse tempo, os grânulos começaram a intumescer aumentando a viscosidade até $3.549,5 \mathrm{cP}(\mathrm{VMAX})$ aos $10,5 \mathrm{~min}$ a $95^{\circ} \mathrm{C}$. A partir da VMAX, o perfil de viscosidade diminuiu até $2.589,5 \mathrm{cP}$ devido ao rompimento dos grânulos liberando amilose. O fenômeno da retrogradação iniciou a partir dos $13 \mathrm{~min}$ em temperatura de $82^{\circ} \mathrm{C}$. A viscosidade elevou-se com o resfriamento da suspensão até o final do experimento, alcançando $6.887,5 \mathrm{cp}$ (VFINAL). Perfil semelhante de viscosidade foi descrito por ZHOU et. al. (2002) no estudo da composição e das propriedades funcionais do arroz e por SACCHETTI et al. (2004) para farinha de arroz cru.

Os perfis de RVA das FME foram organizados em três grupos $\left(E_{1}\right.$ e $E_{6}, E_{2}, E_{10}, E_{15}$ e $E_{16}$; $E_{7}$ a $E_{9}$ e $E_{11}$ a $\left.E_{14}\right)$, representados pelos perfis de viscosidade dos ensaios $E_{3}$ (PVE3), $E_{5}$ (PVE5) e $E_{4}$ (PVE4), respectivamente (Figura 2). 
De modo geral, PVE3 e PVE5 apresentaram pico de VINIC mais agudo e PVE4 mais arredondado com valores maiores que os da farinha de arroz polido, mas menores valores de VMAX e VFINAL. A VINIC foi detectada em tempo curto, entre 1,4 e 3,2 min, comportamento já esperado para material previamente cozido (produto pré-gelatinizado). A forma arredondada do PVE4 indica que o material amiláceo foi menos afetado pelas variáveis do processo de extrusão e que o material prégelatinizado oferece baixa resistência à expansão/ruptura durante 0 tratamento hidrotérmico. Após o pico de VINIC, os perfis (Figura 2) apresentaram padrões diferentes. No PVE5 observou-se perfil mais definido com queda acentuada da viscosidade até alcançar o mínimo entre 13 a 14 min e temperatura de 68 a $76^{\circ} \mathrm{C}$, respectivamente. $\mathrm{O}$ mesmo comportamento ocorreu com PVE3, porém com formação de patamar curto após $7 \mathrm{~min}$. O PVE4 ainda apresentou pico de viscosidade máxima durante o ciclo de aquecimento estabelecido entre 8 a 9 min e 85 a $92^{\circ} \mathrm{C}$. O aparecimento do patamar no PVE3 e o pico de VMAX no PVE4 se deve a existência de grânulos de amido da FAP remanescentes que não sofreram gelatinização durante o processo de extrusão. Por isso ofereceram certa resistência à expansão/ruptura durante o tratamento hidrotérmico aplicado na análise rápida de viscosidade. Durante o ciclo de resfriamento observou-se que todas as pastas apresentaram perfil semelhante, aumentando com o decorrer do tempo de leitura no RVA.

As diferentes formas das curvas de viscosidade das FME observadas (Figura 2) indicam que pelo menos uma das variáveis da extrusão estudadas afetou as características de viscosidade de pasta das farinhas mistas extrusadas.

\subsection{EFEITO DAS VARIÁVEIS DE EXTRUSÃO NAS PROPRIEDADES DE PASTA}

$\mathrm{Na}$ Tabela 2 constam os resultados encontrados para as variáveis dependentes VINIC, VMAX e VFINAL. Como a análise de variância de fator único e o teste de Tukey mostraram diferenças significativas entre as médias (ao nível de $5 \%$ de probabilidade) aplicou-se a análise de variância ao modelo selecionado (Eq. 3), utilizando a MSR, cujos resultados estão apresentados nas Tabelas 3 e 4. 


\section{TABELA 2 - RESULTADOS DO PLANEJAMENTO EXPERIMENTAL EM ESTRELA DA EXTRUSÃO DAS FARINHAS MISTAS EM TERMOS DE VISCOSIDADE INICIAL DE PASTA (VINIC), VISCOSIDADE MÁXIMA (VMAX) E VISCOSIDADE FINAL (VFINAL)}

\begin{tabular}{cclc}
\hline Tratamento & & & \\
\hline 1 & $485,3 \pm 5,8 \mathbf{e}$ & $268,0 \pm 2,6 \mathbf{h i}$ & $374,0 \pm 1,7 \mathbf{g}$ \\
2 & $361,0 \pm 7,2 \mathbf{h}$ & $145,3 \pm 2,5 \mathbf{~ k}$ & $213,3 \pm 4,2 \mathbf{~}$ \\
3 & $665,7 \pm 7,5 \mathbf{~ a}$ & $397,3 \pm 4,0 \mathbf{c}$ & $492,7 \pm 8,1 \mathbf{c}$ \\
4 & $219,0 \pm 2,6 \mathbf{~ k}$ & $357,0 \pm 5,3 \mathbf{d}$ & $459,7 \pm 2,1 \mathbf{d e}$ \\
5 & $632,3 \pm 7,0 \mathbf{b}$ & $256,7 \pm 5,9 \mathbf{i}$ & $367,0 \pm 3,6 \mathbf{g h}$ \\
6 & $426,3 \pm 3,0 \mathbf{f}$ & $215,3 \pm 8,0 \mathbf{j}$ & $272,0 \pm 9,6 \mathbf{k}$ \\
7 & $562,7 \pm 3,2 \mathbf{c}$ & $395,7 \pm 8,3 \mathbf{c}$ & $466,3 \pm 4,0 \mathbf{d}$ \\
8 & $320,7 \pm 7,8 \mathbf{i}$ & $284,3 \pm 3,2 \mathbf{g h}$ & $312,0 \pm 7,5 \mathbf{j}$ \\
9 & $391,0 \pm 6,1 \mathbf{g}$ & $348,0 \pm 6,0 \mathbf{d}$ & $423,3 \pm 4,2 \mathbf{f}$ \\
10 & $645,7 \pm 1,5 \mathbf{b}$ & $313,0 \pm 6,6 \mathbf{e}$ & $379,3 \pm 7,5 \mathbf{g}$ \\
11 & $521,3 \pm 6,5 \mathbf{d}$ & $382,3 \pm 8,3 \mathbf{c}$ & $447,0 \pm 3,0 \mathbf{e}$ \\
12 & $285,0 \pm 7,0 \mathbf{j}$ & $420,3 \pm 4,0 \mathbf{b}$ & $529,7 \pm 2,5 \mathbf{b}$ \\
13 & $416,3 \pm 2,1 \mathbf{f}$ & $447,7 \pm 3,8 \mathbf{a}$ & $583,3 \pm 1,2 \mathbf{~}$ \\
14 & $384,0 \pm 6,6 \mathbf{g}$ & $293,0 \pm 4,6 \mathbf{f g}$ & $343,0 \pm 1,0 \mathbf{i}$ \\
15 & $546,7 \pm 1,5 \mathbf{c}$ & $312,0 \pm 6,0 \mathbf{f}$ & $381,7 \pm 4,0 \mathbf{g}$ \\
16 & $512,3 \pm 6,7 \mathbf{d}$ & $290,3 \pm 5,5 \mathbf{g}$ & $353,0 \pm 4,4 \mathbf{h i}$ \\
\hline
\end{tabular}

Verifica-se pela Tabela 3 que a variação devido à regressão foi significativa estatisticamente ao nível de confiança de $95 \%(p<0,05)$. Os valores dos coeficientes de determinação $\left(\mathrm{r}^{2}\right)$ foram $0,71,0,63 \mathrm{e}$ 0,75 , explicando mais de $60 \%$ da variação total da variável resposta em torno da média e menos de $40 \%$ foram atribuídos aos resíduos. Dos coeficientes da regressão, apenas a variável $R^{2} e$ as variáveis associadas RxFo e TxFo das variáveis independentes VINIC e VMAX, respectivamente, não foram significativas $(p>0,05)$. As equações 
geradas foram:

$\mathrm{VINIC}=527,19+46,81 \cdot \mathrm{R}-39,14 \cdot \mathrm{T}-78,60 \cdot \mathrm{Fo}-39,08 \cdot \mathrm{T}^{2}-40,14 \cdot \mathrm{Fo}^{2}-$ 26,71.R.T + 15,38.R.Fo $-44,79$. T.Fo

VMAX $=309,03-5,46 \cdot R+44,88 \cdot T-42,16 \cdot F o-8,60 \cdot R^{2}+16,44 \cdot T^{2}+5,48 \cdot F^{2}$ $-16,63 . R \cdot T$

VFINAL $=375,67-14,38 \cdot R+47,11 \cdot T-62,03 \cdot F o-8,10 \cdot R^{2}+22,66 \cdot T^{2}+$ 13,76.Fo² $-28,21$.R.T $-6,96$. R.Fo + 8,54.T.Fo

TABELA 3 - ANÁLISE DE VARIÂNCIA PARA O MODELO DE REGRESSÃO QUADRÁTICA DE VISCOSIDADE INICIAL DE PASTA (VINIC), MÁXIMA (VMAX) E FINAL (VFINAL) DAS FARINHAS MISTAS, OBTIDA POR ANÁLISE DE SUPERFÍCIE DE RESPOSTA

\begin{tabular}{|c|c|c|c|c|c|c|c|c|}
\hline & & & & ausas da & arias & & & \\
\hline VINIC & 9 & 62368,31* & 38 & 6029,50 & 5 & $45271,52^{*}$ & 33 & 83,74 \\
\hline VMÁX & 9 & $19924,47^{\star}$ & 38 & 2829,61 & 5 & $21163,29^{\star}$ & 33 & 51,78 \\
\hline VFINAL & 9 & $34127,63^{\star}$ & 38 & 2728,79 & 5 & $20333,87^{\star}$ & 33 & 61,35 \\
\hline
\end{tabular}

* Significativo ao nível de confiança de $95 \%(p<0,05)$. g.I. = graus de liberdade.

Q.M. = quadrado médio.

A VINIC indica a capacidade das farinhas de absorver água em temperatura ambiente e formar pasta, gel ou líquido viscoso como resultado da gelatinização do amido durante o processo de extrusão (BOUVIER, 2001; CARVALHO, ASCHERI e CAL-VIDAL, 2002; FENIMAN, 2004; FERNANDES et al., 2002). Para os coeficientes 
lineares (Tabela 4), a formulação foi a variável com maior influência $(p<0,05)$. Quanto aos coeficientes quadráticos houve influência da formulação e da temperatura. Verificou-se, ainda, que a interação entre as variáveis também foi significativa $(p<0,05)$.

\section{TABELA 4 - ESTIMATIVA DOS COEFICIENTES E EFEITOS DAS VARIÁVEIS DO MODELO POLINOMIAL APLICADO AO PLANEJAMENTO EXPERIMENTAL EM ESTRELA SOBRE AS VARIÁVEIS RESPOSTA VINIC, VMAX E VFINAL}

\begin{tabular}{|c|c|c|c|c|c|c|}
\hline \multirow{3}{*}{ Intercepto } & \multicolumn{6}{|c|}{ Variável independente } \\
\hline & 527,1 & & & & & \\
\hline & & $527,19^{*}$ & 309,03 & $309,03^{*}$ & 375,67 & $375,67^{*}$ \\
\hline $\mathrm{R}$ & 46,81 & $93,62^{*}$ & $-5,46$ & $-10,91^{*}$ & $-14,38$ & $-28,75^{\star}$ \\
\hline $\mathrm{R}^{2}$ & 1,63 & 3,27 n.s. & $-8,60$ & $-17,20^{*}$ & $-8,10$ & $-16,21^{*}$ \\
\hline $\mathrm{T}$ & $-39,14$ & $-78,27^{*}$ & 44,88 & $89,76^{*}$ & 47,11 & $94,22^{*}$ \\
\hline$T^{2}$ & $-39,08$ & $-78,17^{*}$ & 16,44 & $32,88^{*}$ & 22,66 & $45,31^{*}$ \\
\hline Fo & $-78,60$ & $-157,19^{*}$ & $-42,16$ & $-84,32^{*}$ & $-62,03$ & $-124,07^{*}$ \\
\hline $\mathrm{Fo}^{2}$ & $-40,14$ & $-80,29^{*}$ & 5,48 & $10,96^{*}$ & 13,76 & $27,52^{*}$ \\
\hline$R \times T$ & $-26,71$ & $-53,42^{*}$ & $-16,63$ & $-33,25^{\star}$ & $-28,21$ & $-56,42^{*}$ \\
\hline $\mathrm{R} \times \mathrm{Fo}$ & 15,38 & $30,75^{\star}$ & 1,29 & 2,58n.s. & $-6,96$ & $-13,92^{*}$ \\
\hline TxFo & $-44,79$ & $-89,58^{\star}$ & 1,54 & 3,08n.s. & 8,54 & $17,08^{*}$ \\
\hline
\end{tabular}

Coef. $=$ Coeficiente da variável do modelo polinomial da Eq. 3.

* Significativo ao nível de confiança de $95 \%(p<0,05)$.

n.s. = não-significativo.

Na Figura 3 visualiza-se melhor o efeito das variáveis independentes 
no valor de VINIC. Analisando a Figura 3a verifica-se a influência da temperatura e da velocidade do parafuso. A VINIC aumentou com a elevação da velocidade do parafuso e com a temperatura até o valor máximo de $180^{\circ} \mathrm{C}$, aproximadamente, seguido de queda. Com o aumento da temperatura concomitantemente ao da velocidade do parafuso (maior atrito), pontes de hidrogênio que ajudam a manter a integridade da estrutura dos grânulos de amido se rompem facilitando a liberação de amilose para o meio e contribuindo para o aumento do grau de gelatinização. No entanto, altas temperaturas (superiores a $180^{\circ} \mathrm{C}$ ) provocam maior degradação molecular até ocasionar quebra das ligações glucosídicas, formando açúcares com baixo peso molecular que se solubilizam aumentando a viscosidade de pasta no ciclo inicial de aquecimento (CARVALHO, ASCHERI e CAL-VIDAL, 2002; IBANOGLU, AINSWORTH e HAYES, 1996; KOKINI, 1993; LI, CAMPANELLA e HARDACRE, 2004; SACCHETTI et al., 2004).

Segundo a Tabela 2, o maior valor de VINIC foi alcançado pelo $E_{3}(R=$ $100 \mathrm{rpm}, \mathrm{T}=180^{\circ} \mathrm{C}$ e $\left.\mathrm{Fo}=15,0 \%\right)$ e o menor pelo $\mathrm{E}_{12}(\mathrm{R}=150 \mathrm{rpm}, \mathrm{T}$ $=200,45^{\circ} \mathrm{C}$ e Fo $=20,0 \%$ ).

Pela Figura 3b observa-se o efeito positivo de $R$ no valor da viscosidade inicial. Verificou-se que quanto maior a velocidade do parafuso maior será a gelatinização das farinhas mistas e, portanto mais alto será o valor da viscosidade inicial. Entretanto, nas Figuras 3b e 3c, o efeito negativo de maior magnitude da variável independente formulação (Tabela 4) evidenciou que maior proporção de bagaço de jabuticaba diminui o valor da viscosidade inicial das FME. A Tabela 4 mostra o efeito quadrático dessa variável, indicando máxima gelatinização quando combinadas as variáveis $\mathrm{R}=234,09 \mathrm{rpm}$ e $\mathrm{Fo}=20 \%$, com T em $150^{\circ} \mathrm{C}$, correspondendo a $\mathrm{VINIC}=645,7 \mathrm{cP}$ (Tabela 2). A diminuição do valor de VINIC com o aumento da proporção de FBJ na mistura da formulação se deve á menor proporção de material amiláceo na mistura. As fibras incluídas não fornecem suficiente material viscoso fazendo com que a gelatinização do amido diminua (CARVALHO, ASCHERI e CAL-VIDAL, 2002; FERNANDES et al., 2002; FERNANDEZGUTIERREZ et al., 2004; LIN, HSEIH e HUFF et al., 1997; MAGA e FAPOJUWO, 1996 e 1988). 


\section{FIGURA 3 - EFEITO DA EXTRUSÃO NO VALOR DE VINIC DAS PASTAS DE FME DE ARROZ POLIDO E BAGAÇO DE JABUTICABA: a) ROTAÇÃO DO PARAFUSO VS. TEMPERATURA, b) ROTAÇÃO DO PARAFUSO VS. FORMULAÇÃO E, c) TEMPERATURA VS. FORMULAÇÃ̃O}

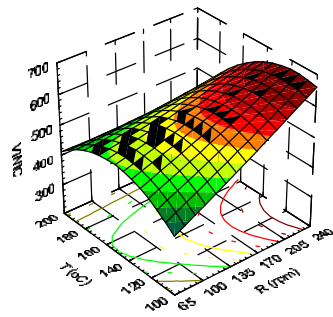

(a)

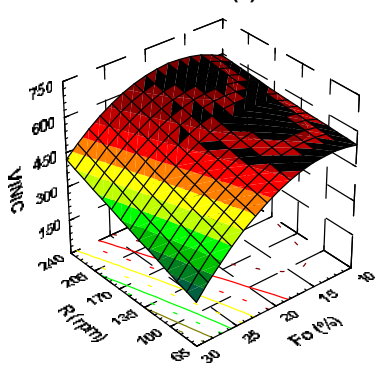

(b)

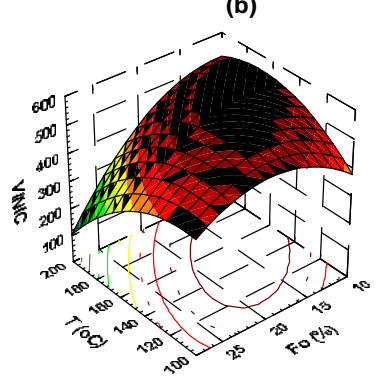

(c)


As Figuras 4 e 5 demonstram o efeito das variáveis independentes no valor de VMAX e VFINAL, respectivamente. As Figuras $4 \mathrm{a}$ e 5a, $4 \mathrm{~b}$ e $5 b$ e $4 c$ e $5 c$ revelaram perfis de superfície de resposta semelhantes. 
Assim, a viscosidade independentemente da variável estudada aumentou com a temperatura e diminuiu com a porcentagem de FBJ nas FME. Além disso, pouca variação desses valores foi observada com as mudanças na velocidade do parafuso.

FIGURA 4 - EFEITO DAS VARIÁVEIS DE EXTRUSÃO NA VMAX DE FME DE ARROZ POLIDO E BAGAÇO DE JABUTICABA: a) ROTAÇÃO DO PARAFUSO VS. TEMPERATURA, b) ROTAÇÃO DO PARAFUSO VS. FORMULAÇÃO E c) TEMPERATURA VS. FORMULACÃO

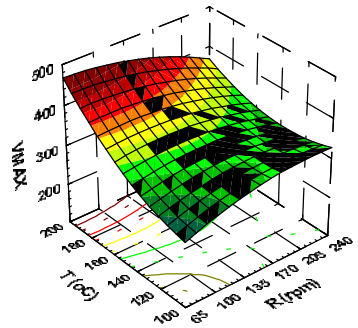

(a)

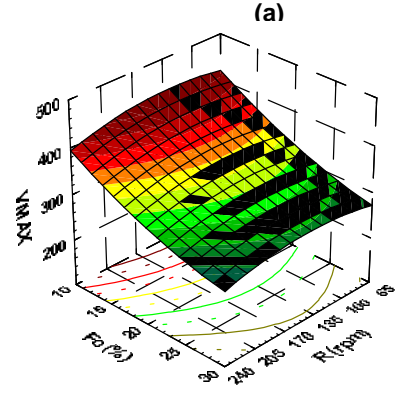

(b)

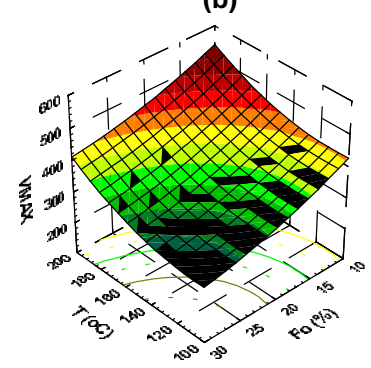

(c)
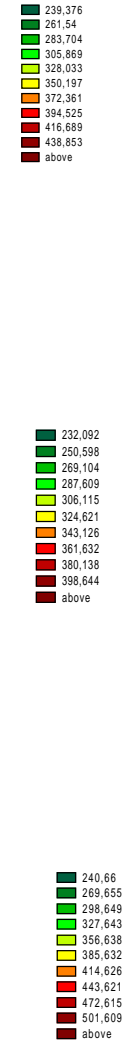
Segundo CARVALHO, ASCHERI e CAL-VIDAL (2002), a VMAX mede a resistência do grânulo de amido ao colapso resultante da ação da temperatura e do atrito mecânico, promovido durante a determinação de viscosidade de pasta pelo RVA. Durante o ciclo de aquecimento da FAP até $95^{\circ} \mathrm{C}$ (Figura 1b) ocorreu intumescimento e gelatinização dos grânulos de amido, atingindo o pico máximo nessa etapa. Em processamento mais severo por extrusão, a estrutura cristalina do amido pode ser totalmente destruída de tal forma que no ciclo de aquecimento o perfil de viscosidade do material amiláceo pode revelar ausência de pico com viscosidade baixa. Em condições brandas o material amiláceo poderá conservar parte da integridade dos grânulos de amido, observando-se no perfil de viscosidade valores relativamente altos de VMAX. De acordo com esses autores e com a Figura 2 as condições do processo de extrusão sofridas pelos ensaios representados pelo $E_{5}$ foram severas, uma vez que o PVE5 não apresentou pico de viscosidade máxima. Ao contrário, o pico de viscosidade máxima do $\mathrm{PVE}_{4}$ indicou que o processo de extrusão foi mais brando. Na Figura 4, considerando os efeitos quadráticos das variáveis independentes, as condições brandas e severas podem ser obtidas combinando as variáveis $R=150 \mathrm{rpm}, T=200,41^{\circ} \mathrm{C}$ e $\mathrm{Fo}=11,59 \%$ e $\mathrm{R}=150 \mathrm{rpm}, \mathrm{T}=99,55^{\circ} \mathrm{C}$ e Fo $=28,41 \%$, com $\mathrm{VMAX}$ igual a 513,08 cP e 220,28 cP, respectivamente. Assim, quanto maior a temperatura e menor proporção de fibra alimentar na mistura das farinhas extrusadas, maior o pico de viscosidade máxima. Já quanto maior a presença dessas fibras e mais baixa a temperatura menor é o pico de viscosidade máxima.

Sabe-se que durante o ciclo de resfriamento, as pastas de amido normalmente tendem à retrogradação implicando no aumento da viscosidade de pasta a $25^{\circ} \mathrm{C}$ (VFINAL). Pela Tabela 4 verifica-se que os coeficientes lineares de temperatura e de formulação, o coeficiente quadrático para a temperatura, bem como a interação velocidade do parafuso e temperatura apresentaram maiores efeitos sobre a VFINAL. Nota-se ainda que a variável formulação exerce maior efeito $(p<0,05)$ e foi inversamente proporcional à viscosidade, enquanto que a temperatura com seu efeito positivo aumentou linear e quadraticamente a VFINAL. No entanto, a interação das variáveis $R \times T$ foi negativa $(p<0,05)$. A Figura 5 mostra o efeito dessas variáveis sobre as farinhas mistas extrusadas de arroz polido de bagaço de jabuticaba. A Figura 5 a indica maior influência da temperatura, pois com $100^{\circ} \mathrm{C}$ a $120^{\circ} \mathrm{C}$ e $\mathrm{R}$ variando 
de 65,9 rpm a 234,10 rpm a viscosidade aumenta. Em temperatura acima de $120^{\circ} \mathrm{C}$, mantendo-se a mesma velocidade do parafuso a viscosidade de pasta aumenta. Entretanto, em temperaturas maiores que $150^{\circ} \mathrm{C}$ e $\mathrm{R}$ variando de 65,9 a $234,10 \mathrm{rpm}$, a viscosidade diminui.

\section{FIGURA 5 - EFEITO DAS VARIÁVEIS DE EXTRUSÃO NA VFINAL DE FME DE ARROZ POLIDO E BAGAÇO DE JABUTICABA: a) ROTAÇÃO DO PARAFUSO VS. TEMPERATURA, b) ROTAÇÃO DO PARAFUSO VS. FORMULAÇÃO E c) TEMPERATURA VS. FORMULAÇÃ̃O}

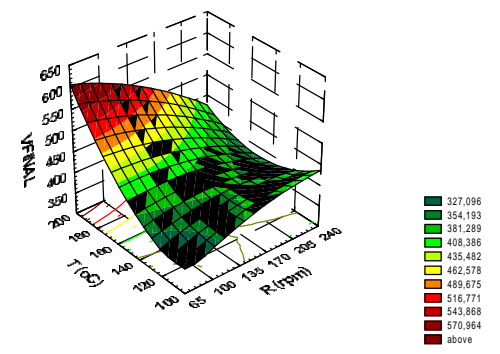

(a)



(b)

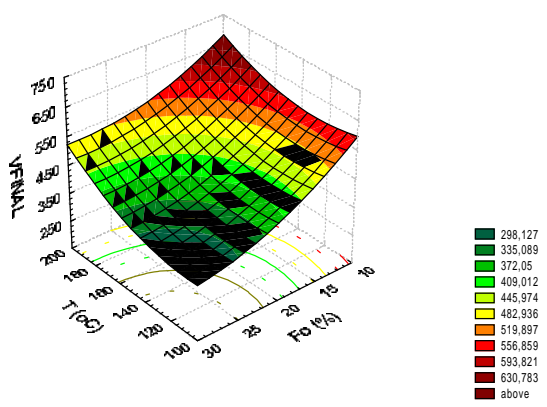

(c) 
$\mathrm{Na}$ Figura $5 \mathrm{~b}$ verifica-se o efeito das variáveis formulação e velocidade do parafuso nas características de pasta no ciclo de resfriamento. Observou-se que maior teor de bagaço na formulação diminui a viscosidade a $25^{\circ} \mathrm{C}$. Os menores valores de VFINAL ocorreram com adição de farinha de bagaço de jabuticaba acima de $25 \%$. Nessa faixa de porcentagem de FBJ notou-se que quando $R$ varia de 65,9 a 234,10 rpm a VFINAL diminui. Em porcentagens menores de FBJ (abaixo de $25 \%$ ) a viscosidade a $25^{\circ} \mathrm{C}$ aumentou em todas as velocidades do parafuso, confirmando maior influência da variável formulação.

A Figura $5 c$ evidencia que o valor da VFINAL diminui com o aumento da porcentagem de FBJ na mistura independentemente da temperatura e que aumenta com a elevação da temperatura, independentemente da formulação. Experimentalmente, os valores máximo e mínimo foram alcançados nas combinações das variáveis $R=150 \mathrm{rpm}, T=200,46^{\circ} \mathrm{C}$ e $\mathrm{Fo}=11,59 \%$ e $\mathrm{R}=150 \mathrm{rpm}, \mathrm{T}=99,54^{\circ} \mathrm{C}$ e $\mathrm{Fo}=28,41 \%$, respectivamente.

\section{CONCLUSÃO}

De acordo com a análise de fibra detergente neutro, as farinhas mistas pré-gelatinizadas de arroz polido e de bagaço de jabuticaba podem ser consideradas como boa fonte de fibra alimentar conforme a legislação brasileira.

As amostras estudadas apresentaram perfis diferentes de viscosidade com valores mínimos para a farinha de bagaço de jabuticaba, máximos para a farinha de arroz polido e valores de viscosidade intermediários para as amostras de farinha mista extrusada. A forma e o valor do pico da viscosidade inicial e da máxima indicaram que as condições do processo de extrusão afetaram as propriedades de pasta das farinhas mistas de arroz polido e de bagaço de jabuticaba.

A viscosidade inicial de pasta a $25^{\circ} \mathrm{C}$ foi fortemente influenciada pela formulação e pela velocidade do parafuso, seguidas pela temperatura. A viscosidade inicial de pasta mostrou-se inversamente proporcional à porcentagem de fibra alimentar adicionada à formulação de farinhas mistas de arroz polido e bagaço de jabuticaba. Também revelou-se 
inversamente proporcional à temperatura do processo de extrusão, porém diretamente proporcional à velocidade do parafuso.

A viscosidade máxima e a viscosidade final de pasta foram bastante influenciadas pela formulação e pela temperatura, seguidas pela velocidade do parafuso. Ambas (VMAX e VFINAL) aumentaram com a elevação da temperatura e diminuíram com o aumento da porcentagem de FBJ na mistura, bem como com o incremento da velocidade de rotação do parafuso.

\begin{abstract}
PRODUCTION OF PRE-GELATINIZED FLOURS FROM RICE AND JABUTICABA BAGASSE: EFFECT OF EXTRUSION VARIABLES ON THE PASTE PROPERTIES

Central composite design and mathematical model response surface methodology were used to study the effect of partial substitution of polished rice flour (PRF) for jabuticaba bagasse flour (JBF) as fiber source $(11.59-28.41 \%)$, considering levels of screw speed $(65.9-234.1 \mathrm{rpm})$ and final extruder heating zone temperatures $\left(99.54-200,46^{\circ} \mathrm{C}\right)$. The acid detergent fiber (ADF) and the neutral detergent fiber (NDF) of raw material flours and their mistures were determined. The paste viscosity property was carried out in Rapid Visco Analyzer (RVA). JBF showed high value of ACD and NDF, 21.91 and $45.47 \%$, respectively, which contributed to increase the fiber content of the final extruded flours (FEF). The paste viscosity profile varied from very low viscosity for the JBF and higher viscosity for the PRF samples, being FEF with an intermediate paste viscosity. The initial paste viscosity at $25^{\circ} \mathrm{C}$ was strongly affected by formulation and screw speed followed by temperature. The maximum peak at $95^{\circ} \mathrm{C}$ (VMAX) and the final viscosity (VFINAL) were affected by formulation, temperature and screw speed. Both increased with higher temperatures, and reduced due the addition of JBF in the mixture and as the screw speed increased.
\end{abstract}

KEY-WORDS: THERMOPLASTIC EXTRUSION; RICE FLOUR; DIETARY FIBER; JABUTICABA.

\title{
REFERÊNCIAS
}

1 AKDOGAN, $\mathrm{H}$. High moisture food extrusion. International Journal of Food Science and Technology, v. 34, p. 195-207, 1999.

2 AKDOGAN, H. Pressure, torque, and energy responses of a twin screw extruder at high moisture contents. Food Research International, v. 29, n. 5-6, p. 423-429, 1996. 
3 AACC. American Association of Cereal Chemists. Approved methods of the American Association of Cereal Chemists. $8^{\text {th }}$ ed. St. Paul, 1995.

4 AMES, J., M.; DEFAYE, A. B.; BAILEY, R. G.; BATES, L. Analysis of the non-volatile Maillard reaction products formed in an estrusion-cooked model food system. Food Chemistry, v. 61, n. 4, p. 521-524, 1998.

5 ANTONIO, X., M. de. La fibra en la alimentación. Farmacia hospitalaria. Barcelona: Edikamed S. L., 2004. 27 p.

6 BALAGOPALAN, C. Cassava utilization in food, feed and industry. In: HILLOCKS, R. J.; THRESH, J. M.; BELLOTTI, A. C. Cassava: biology, production and utilization. Wallingford: CAB International, 2002. p. 301-317.

7 BHANDARI, B.; D'ARCY, B.; YOUNG, G. Flavour retention during high temperature short time extrusion cooking process: a review. International Journal of Food Science and Technology, v. 36, p. 453461, 2001.

8 BOONYASIRIKOOL, P.; CHARUNUCH, C. Development of nutritious soy fortified snack by extrusion Cooking. Kasetsart J. (Nat. Sci.), v. 34, n. 3, p. 355-365, 2000.

9 BOTELHO, L.; CONCEIÇÃO, A.; CARVALHO, V. D. Caracterização de fibras alimentares da casca e cilindro central do abacaxi 'smooth cayenne'. Ciênc. Agrotec., v. 26, n. 2, p. 362-367, 2002.

10 BOUVIER, J. M. Breakfast cereals. In: GUY, R. Extrusion cooking. Boca Raton: Woodhead Publishing, 2001. p. 133-160.

11 BOX, G. E. P.; HUNTER, W. G.; HUNTER, J. S. Statistics for experimenters: an introduction to design, data analysis, and model building. New York: Wiley and Sons, 1978. 653 p.

12 BRASIL. Portaria n.27 de 13 de janeiro de 1998. Regulamento técnico referente à informação nutricional complementar. Diário Oficial [da] República Federativa do Brasil. Disponível: http://e-legis.bvs.br/leisref/ public/showAct.php?id=97. Acesso em: $10 \mathrm{dez}$. 2004.

13 CAMIRE, M. E. The definition of dietary fiber. Cereal Foods World, v. 46, n. 3, p. 112-124, 2001. 
14 CARVALHO, R. V.; ASCHERI, J. R. A.; CAL-VIDAL, J. Efeito dos parâmetros de extrusão nas propriedades físicas de pellets $(3 \mathrm{~g})$ de misturas de farinhas de trigo, arroz e banana. Ciências Agrotécnicas, v. 26, n. 5, p. 1006-1018, 2002.

15 CHEYNE, A.; BARNES, J.; WILSON, D. I. Extrusion behaviour of cohesive potato starch pastes: I. Rheological characterisation. Journal of Food Engineering, v. 66, p. 1-12, 2005.

16 CHIANG, B. Y.; JOHNSON, J. A. Gelatinization of starch in extruded products. Cereal Chemistry, v. 54, n. 3, p. 436-443, 1977.

17 CHUANG, G. C. C.; YEH, A. I. Effect of screw profile on residence time distribution and starch gelatinization of rice flour during single screw extrusion cooking. Journal of Food Engineering, v. 63, p. 21-31, 2004.

18 COCHRAN, B. Y.; COX, G. M. Experimental desings. $2^{\text {nd }}$ ed. New York: John Willey, 1964.

19 COLONNA, P.; DSOUBLIER, J. L.; MELCION, J. P.; MONREDON, F. de; MERCIER, C. Extrusion cooking and drum drying of wheat starch. I. Physical and macromolecular modifications. Cereal Chem., v. 61, p. 538-543, 1984.

20 DING, Q. B.; AINSWORTH, P.; TUCKER, G.; MARSON, H. The efect of extrusion conditions on the physicochemical properties and sensory characteristics of rice-based expanded snacks. Journal of Food Engineering, v. 66, p. 283-289, 2005.

21 DREHER, M. L. Food industry perspective: functional properties and food uses of dietary fiber. In: KRITCHEVSKY, D.; BONFIELD, C, (ed). Dietary fiber in health \& disease. Minnesota: Eagan Press, 1995. p. 467-74.

22 FENIMAN, Cristiane Mengue. Caracterização de raízes de mandioca (Manihot sculenta, Crantz) do cultivar IAC 576-70 quanto à cocção, composição química e propriedades do amido em duas épocas de colheita. Piracicaba, 2004, 83 p. Tese (Mestrado em Ciências), Escola Superior de Agricultura "Luiz de Queiroz", Universidade de São Paulo.

23 FERNANDES, M. S.; WANG, S. H.; ASCHERI, J. L. R.; OLIVEIRA, M. F.; COSTA, S. A. J. Produtos extrusados expandidos de misturas de canjiquinha e soja para uso como petiscos. Pesquisa Agropecuária Brasileira, v. 37, n. 10, p. 1495-1501, 2002. 
24 FERNÁNDEZ-GUTIÉRREZ, J. A.; SAN MARTÍN-MARTÍNEZ, E.; MARTÍNEZ-BUSTOS, F.; CRUZ-OREA, A. Physicochemical properties of casein-starch interaction obtained by extrusion process. Starch/ Stärke, v. 56, p. 190-198, 2004.

25 FUKUOKA, M.; OHTA, K. I.; WATANABE, H. Determination of the terminal extent of starch gelatinization in a limited water system by DSC. Journal of Food Engineering, v. 53, n. 1, p. 39-42, 2002.

26 GARCÍA-LUNA, I. N. Caracterización fisicoquímica y funcional de los residuos fibrosos de mango criollo (Mangifera indica $L$ ) y su incorporación en galletas. Huajuapan de León, 2003, 50 p. Tese (Doutorado em Ingeniería de Alimentos), Facultad de Ingeniería de Alimentos, Universidad Tecnológica de la Mixteca.

27 GUY, R. Raw materials for extrusion cooking. In: GUY, R. Extrusion cooking. Boca Raton: Woodhead Publishing, 2001a. p. 5-28.

28 GUY, R. Snack foods. In: GUY, R. Extrusion cooking. Boca Raton: Woodhead Publishing, 2001b. p. 161-181.

29 HSIEH, S. L.; HUFF, H. E. Effects of lipids and processing conditions on degree of starch gelatinization of extruded dry pet food. Lebensm.Wiss. u.-Technol., v. 30, n. 7, p. 754-761, 1997.

30 IBANOGLU, S.; AINSWORTH, P.; HAYES, G. D. Extrusion of tarhana: effect of operating variables on starch gelatinization. Food Chemistry, v. 51, n. 4, p. 541-544, 1996.

31 ILO, S.; BERGHOFER, E. Kinetics of colour changes during extrusion cooking of maize grits. Journal of Food Engineering, v. 39, p. 73-80, 1999.

32 IWE, M. O.; Van ZUILICHEM, D. J.; STOLP, W.; NGODDY, P. O. Effect of extrusion cooking of soy-sweet potato mixtures on available lysine content and browning index of extrudates. Journal of Food Engineering, v. 62, p. 143-150, 2004.

33 KARAPANTSIOS, T.D.; SAKONIDOU, E.P.; RAPHAELIDES, S. N. Water dispersion kinetics during starch gelatinization. Carbohydrate Polymers, v. 49, n. 4, p. 479-490, 2002.

$34 \mathrm{KOKINI}, \mathrm{J}$. L. The effect of processing history on chemical changes in single- and twin-screw extruders. Trends Food Sci. Technol., v. 4, 
p. 324-329, 1993.

35 KOKINI, J. L.; HO, C. T.; KARWE, M. V. Food extrusion science and technology. New York: Marcel Dekker, 1992.

36 LAI, L. S.; KOKINI, J. L. Estimation of viscous heat effects in slit flow of 98\% amylopectin (Amioca), 70\% amylose (Hylon 7) corn starches and corn meal during extrusion. J. Food Eng., v. 16, p. 309-318, 1992.

37 LAWTON, B. J.; HENDERSON, G. A.; DERLATSKA, E. J. The effects of extruder variables on the gelatinization of corn starch. Canadian Journal of Chemical Engineering, v. 50, n. 4, p. 168-172, 1972.

38 LI, P. X.; CAMPANELLA, O. H.; HARDACRE, A. K. Using an in-line slitdie viscometer to study the effects of extrusion parameters on corn melt rheology. Cereal Chemistry, v. 81, n. 1, p. 70-76, 2004.

39 LIN, S.; HSEIH, F.; HUFF, H. E. Effects of lipids and processing conditions on degree of starch gelatinization of extruded dry pet food. Lebens Wiss Technol, v. 30, p. 754-61, 1997.

40 LOBO, A. R.; SILVA, G. M. de L. Amido resistente e suas propriedades físico-químicas. Rev. Nutr., v. 16, n. 2, p. 219-226, 2003.

41 MAGA, J. A.; FAPOJUWO, O. O. Extrusion of corn grits containing various levels of hydrocolloids. Journal of Food Technology, v. 21, p. 61-66, 1986.

42 MAGA, J. A.; FAPOJUWO, O. O. The effect of the various hydrocolloids on some physical properties of extruded corn grits. Int. J. Food Sci. Technol., v.23, p. 49-56, 1988.

43 MATUDA, Tatiana Guinoza. Análise térmica da massa de pão francês durante os processos de congelamento e descongelamento: otimização de uso de aditivos. São Paulo, 2004.142 p. Tese (Doutorado em Engenharia), Escola Politécnica da Universidade de São Paulo, Universidade de São Paulo.

44 MITCHELL, J.R.; HILL, S.E.; PATERSON, L.; VALLÈS, B.; BARCLAY, F.; BLANSHARD, J.M.V. The role of molecular weight in the conversion of starch. In: FRAZIER, P.J.; DONALD, A.M.; RICHMOND, P. (Eds.) Starch structure and functionality. London: The Royal Chemistry Society, 1997. pp. 69-76. 
45 MUNHOZ, M. P.; WEBER, F. H.; CHANG, Y. K. Influência de hidrocolóides na textura de gel de amido de milho. Ciênc. Tecnol. Aliment., v. 24, n. 3, p. 403-406, 2004

46 ØSTERGÅRD, K.; BJÖRK, I.; VAINIONPÅÅ, J. Effects of extrusion cooking on starch and dietary fiber in barley. Food Chemistry, v. 34, p. 215-227, 1989.

47 PIMENTEL-GOMES, F. P. Curso de estatística experimental. 12. ed. São Paulo: ESALQ/USP, 2000. 467 p.

48 POURCHET-CAMPOS, M.A. Fibra e nutrição. Boletim da Sociedade Brasileira de Ciência e Tecnologia de Alimentos, v.22, n. 3/4, p. 167171, 1988.

49 SACCHETTI G.; PINNAVAIA, G. G.; GUIDOLIN, E.; DALLA ROSA, M. Effects of extrusion temperature and feed composition on the functional, physical and sensory properties of chestnut and rice flour-based snacklike products. Food Research International, v. 37, p. 527-534, 2004.

50 SOUZA, G. B. de; NOGUEIRA, A. R. de A.; SUMI, L. M.;BATISTA, L. A. R. Método alternativo para a determinação de fibra em detergente neutro e detergente ácido. São Carlos: Embrapa Pecuária Sudeste. (Boletim de Pesquisa, 4), 1999. 21 p.

51 STATSOFT, INC. Statistica for Windows (computer program manual). Tulsa, OK, 1996. 1 CD-ROM.

52 THIRÉ, R. M. S. M.; SIMÃO, R. A.; ANDRADE, C. T. High resolution imaging of the microstructure of maize starch films. Carbohydrate Polymers, v. 54, n. 2, p. 149-158, 2003.

53 TUNGLAND, B. C.; MEYER, D. Nondigestible oligo- and polysaccharides (dietary fiber): their physiology and role in human health and food. Comprehensive Reviews in Food Science and Food Safety, v. 3, p. 73-92, 2002.

54 VAN DEN EINDE, R. M.; VAN DER VEEN, M. E.; BOSMAN, H., VAN DER GOOT, A. J.; BOON, R. M. Modeling macromolecular degradation of corn starch in a twin screw extruder. Journal of Food Engineering, v. 66, p.147-154, 2005.

55 VAN LENGERICH, B. Influence of extrusion processing on in-line rheologycal behavior, structure, and function of wheat starch. In: FARIDI, 
H.; FAUBION, J. M. Dough rheology and baked product texture. New York: Van Nostrand Reinhold, 1990. p. 421.

56 VERGNES, B.; DELLA VALLE, G.; TAYEB, J. A specific alit die rheometer for extruded starchy products. Design, validation and application to maize starch. Rheol. Acta, v. 32, p. 465-476, 1993.

57 ZHOU, Z.; ROBARDS, K.; HELLIWELL, S.; BLANCHARD, C. Composition and functional properties of rice. International Journal of Food Science and Technology, v. 37, p. 849-868, 2002.

\section{Agradecimentos}

Os autores agradecem ao Instituto de Macromoléculas Prof ${ }^{\mathrm{a}}$. Eloísa Mano da Universidade Federal do Rio de Janeiro (IMA/UFRJ). À Fazenda Jabuticabal do Município de Nova Fátima do Estado de Goiás pela matériaprima concedida. 